\title{
Recurrent pneumonia in a 38-year-old female
}

\section{Case history}

A 38-year-old Japanese female was referred to Kurume University Hospital (Kurume, Japan) because of recurrent pneumonia. She had no particular medical history, had never undergone a blood transfusion and was taking no regular medication or treatment. She had a negative smoking history, did not own any pets and denied a history of dust exposure. Her family history was unremarkable. She had successfully completed three vaginal deliveries and had been well until the age of 35 years, which is when she experienced her first episode of pneumonia. The pneumonia was cured by the administration of $\beta$ lactam antibiotics. She then suffered three more episodes of pneumonia within 3 years. She denied any history of accidental aspiration, weight loss or
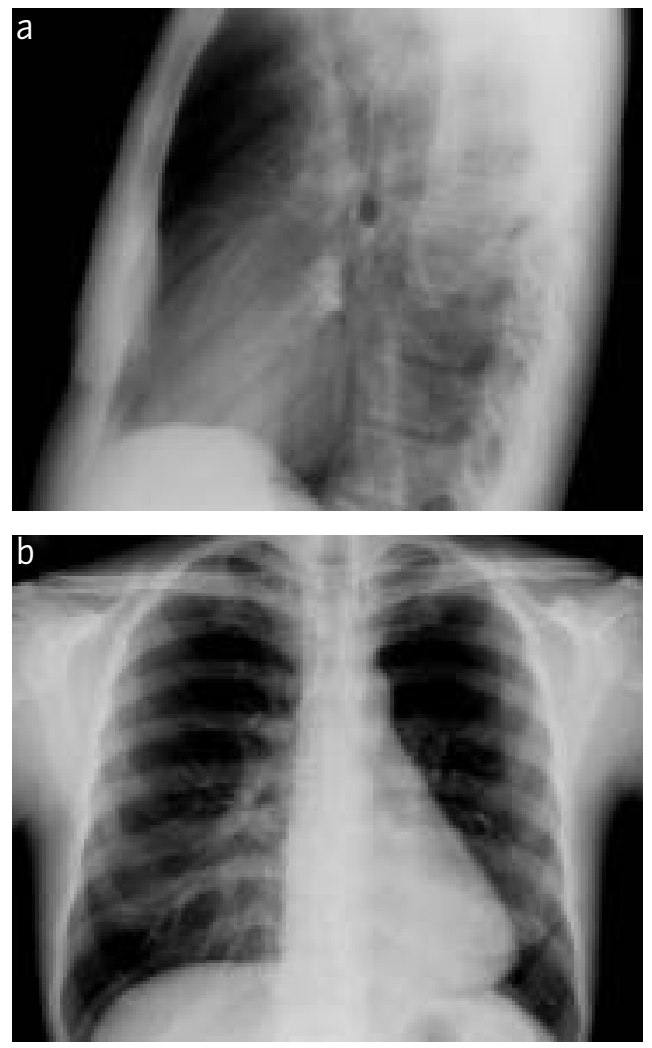

Figure 1

Chest radiographs at admission: a) posteroanterior projection, and b) left lateral projection. haemoptysis, and did not have pyrexia, night sweats or sputum production between the episodes of pneumonia.

The patient was asymptomatic on referral. The result of physical examination was not particular. A complete blood count, blood chemistry for liver and renal function, electrolytes and blood sugar were all within the normal limits. The erythrocyte sedimentation rate was $17 \mathrm{~mm}$ per hour. Serum complements $(C 3, C 4)$ and immunoglobulins $A, M$ and $G$ were within the normal range. Finally, a test for HIV was negative.
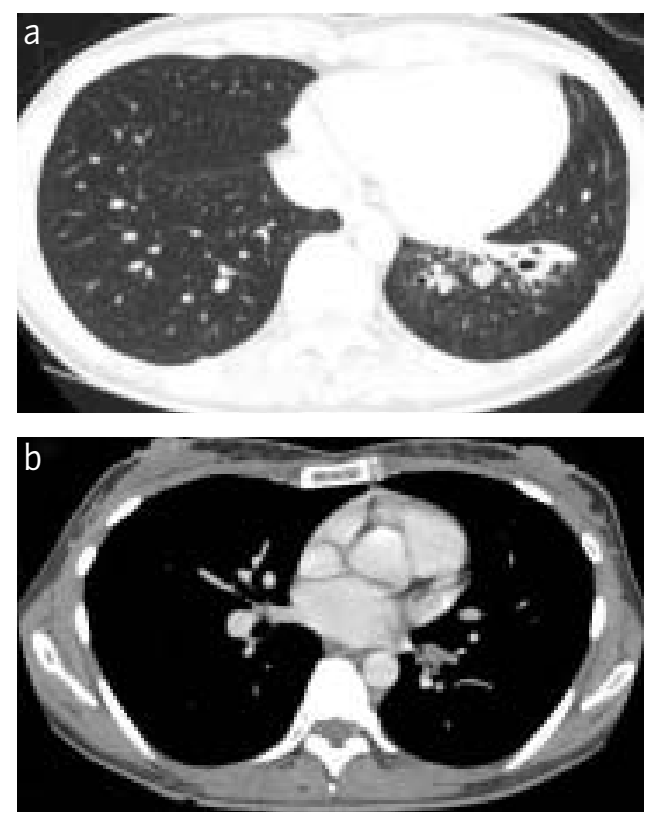

Figure 2

CT scans at admission.

\section{Task 1}

Interpret the chest radiographs and CT scans presented.
T. Koga $a^{1}$

J. Ikeda ${ }^{1}$

K. Fujimoto ${ }^{2}$

S. Takamori ${ }^{3}$

M. Kinoshita

H. Aizawa ${ }^{1}$

${ }^{1}$ First Dept of Internal Medicine, ${ }^{2}$ Dept of Radiology, and ${ }^{3}$ Dept of Surgery, Kurume University School of Medicine, Kurume, Japan.

\section{Correspondence: \\ T. Koga \\ 67 Asahimachi \\ Kurume \\ 830-0011 Japan \\ Fax: 81942317703 \\ E-mail: \\ kogat@med.kurume-u.ac.jp}




\section{Answer 1 \\ The posteroanterior projection (figure 1a) revealed reduced translucency of the left lower lung field. Upper lobar bronchial radiolucency was dislocated downwards and the lower lobar bronchus in the left lung was truncated. Left lateral projection (figure $1 \mathrm{~b}$ ) revealed band opacity in the dorsobasal portion of the left lung. No left upper lobar bronchial orifice was discernible. \\ The computed tomography (CT) scans (thin section) of the thorax showed centrilobular ground-glass opacities in the left basal seg- ments and disclosed ecstatic left basal bronchi (figure 2a), which was originally obstructed by an endobronchial tumour (figure $2 \mathrm{~b}$ ).}

\section{Task 2}

At this stage, which further diagnostic procedures would you recommend?

\section{Answer 2}

Fibreoptic bronchoscopy and left lower lobectomy were performed

\section{Diagnostic procedures}

\section{Fibreoptic bronchoscopy}

At this point, an endobronchial tumour in the left basal bronchus was suspected. Bronchogenic carcinoma occurs as frequently as endobronchial tumour; however, it was assumed that the tumour was more likely to be benign in this case, i.e. benign neoplastic or inflammatory tumour. Subsequently, fibreoptic bronchoscopy was performed and disclosed a whitish tumour with a smooth surface obstructing the left basal bronchus (figure 3). Biopsy yielded a normal bronchial epithelial layer with fibrosis underneath and was not conclusive.

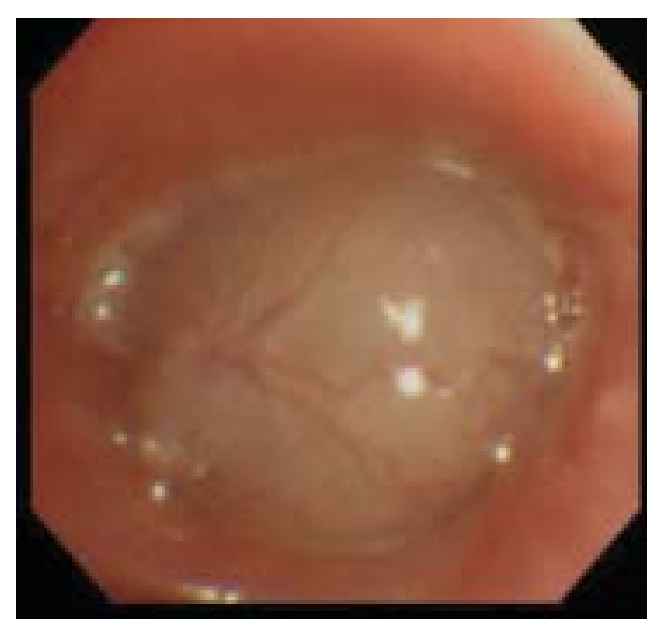

Figure 3

Fibreoptic bronchoscopy showing a whitish tumour with a smooth surface obstructing the left basal bronchus. 


\section{Left lower lobectomy}

Due to the tumour size and the extended bronchiectasis, surgical left lower lobectomy was selected as the treatment for this patient. Figure 4 displays the excised left lung. There was a welldemarcated whitish tumour obstructing the left lower lobar bronchus (arrow). Microscopically, the luminal surface of the tumour was circumscribed with an intact epithelial layer (figure 5a), consistent with the bronchoscopic findings. The tumour was characterised by spindle cell proliferation, arranged in fascicles, cigar-shaped blunted nuclei, eosinophilic cytoplasm and no significant mitotic activity or pleomorphism (figure $5 b$ ). Immunostaining gave a diffuse positive result for smooth muscle actin (figure 5c).

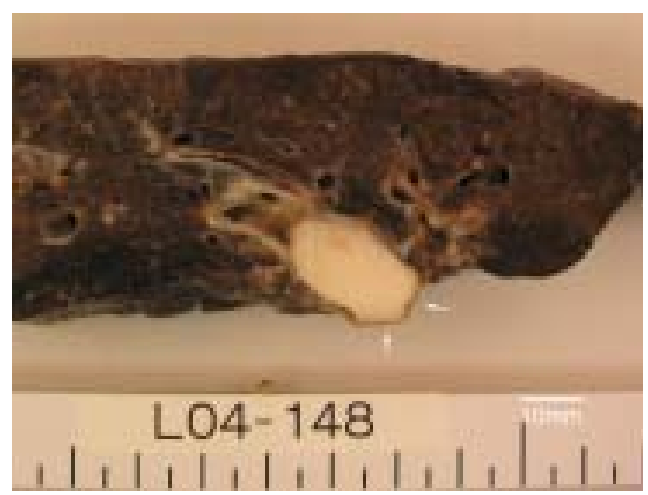

Figure 4

The excised left lung. a
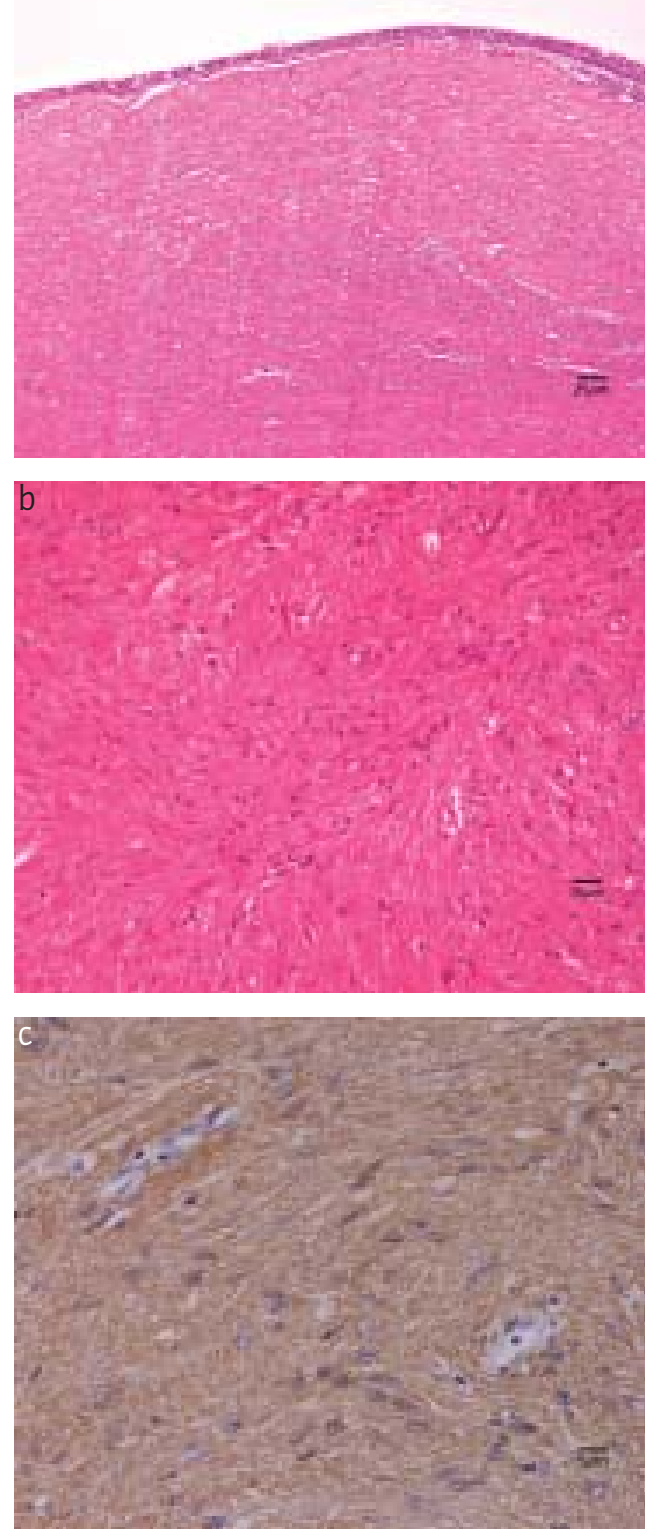

Figure 5

Microscopic findings.

Internal scale bar:

a) $25 \mu \mathrm{m}$;

b) $10 \mu \mathrm{m}$

c) $5 \mu \mathrm{m}$.

Task 3

Based on the patient history, chest radiographs, CT scans, lobectomy and bronchoscopy findings, what would your diagnosis be? 


\section{Answer 3 \\ A diagnosis of bronchial leiomyoma and sec- ondary bronchiectasis was made.}

\section{Discussion}

The present case is characterised by recurrent pneumonia in a patient without underlying conditions that predispose to respiratory infections. Recurrent pneumonia is frequently associated with pathological conditions, such as immunodeficiency in adults [1] and congenital anomalies in children [2]. Disorders associated with deranged local immunity, such as bronchiectasis, bronchial foreign bodies [3] or tumours [4], are also frequent causes of recurrent pneumonia. Particularly, recurrent pneumonia occurring in the same anatomical location is suggestive of endobronchial tumour. In this sense, the latter is relevant to the present case because of the radiological findings of the lung: the chest radiograph and CT scans of the thorax indicated an endobronchial tumour and, presumably, secondary bronchiectasis. As a matter of fact, bronchoscopy identified an endobronchial tumour with a smooth surface obliterating the left basal bronchus in this patient.

Carcinoid tumour accounts for $1-2 \%$ of all lung tumours, and frequently affects large airways such as lobar and segmental bronchi. Although there are aggressive variants, carcinoid tumour of low-grade malignancy can take indolent clinical presentations such as recurrent pneumonia, as in the present case. SHAH et al. [5] have previously reviewed $\sim 4,000$ patients with endobronchial tumours and found that benign ones accounted for $<5 \%$, the majority of which were papilloma, hamartoma and chondrohamartoma. Leiomyoma was responsible for only $3 \%$ of those benign tumours [5]. Leiomyoma of the lung affects lung parenchyma [6], as well as proximal airways [7], as in the present case, and produces various clinical pictures depending on the location and size of the tumour. Chronic or repeated pneumonia or bronchiectasis, as in the present case, can occur if the tumour obstructs conducting airways [7]. The treatment of choice for leiomyoma appears to be surgical excision [7]. Although less invasive procedures, such as endoscopic tumour resection, have been successfully applied in selected patients [8], surgical left lower lobectomy was selected in the present case because of the size of the tumour and the bronchiectasis, which would predispose the patient to recurrent pneumonia or haemoptysis if it remained.

In mostcases, the diagnosis of leiomyoma can be established by light microscopy, and immunochemical stains can be of help in supporting the diagnosis. The most important diagnostic consideration may be assessing the malignant potential of the tumour, which is suspected by high mitotic activity, nuclear pleomorphism, necrosis and haemorrhage. Recurrence of bronchial leiomyoma is not common.

\section{References}

1. Ekdahl K, Braconier JH, Rollof J. Recurrent pneumonia: a review of 90 adult patients. Scand J Infect Dis 1992; 24: 71-76.

2. Owayed AF, Campbell DM, Wang EE. Underlying causes of recurrent pneumonia in children. Arch Pediatr Adolesc Med 2000; 154: 190-194.

3. Blazer S, Naveh Y, Friedman A. Foreign body in the airway. A review of 200 cases. Am J Dis Child 1980; 134: 68-71.

4. Xu LT, Sun ZF, Li ZJ, Wu LH, Wang ZZ. Tracheobronchial tumors: an eighteen-year series from Capital Hospital, Peking, China. Ann Thorac Surg 1983; 35: 590-596.

5. Shah H, Garbe L, Nussbaum E, Dumon JF, Chiodera PL, Cavaliere S. Benign tumors of the tracheobronchial tree. Endoscopic characteristics and role of laser resection. Chest 1995; 107: 1744-1751.

6. Yellin A, Rosenman Y, Lieberman Y. Review of smooth muscle tumours of the lower respiratory tract. Br J Dis Chest 1984; 78: 337-351.

7. Vera-Roman JM, Sobonya RE, Gomez-Garcia JL, Sanz-Bondia JR, Paris-Romeu F. Leiomyoma of the lung. Cancer 1983; 52: 936-941.

8. Kim KH, Suh JS, Han WS. Leiomyoma of the bronchus treated by endoscopic resection. Ann Thorac Surg 1993; 56: 1164-1166. 